Seiko Hamada Nobuaki Shime

\section{Emphysematous pancreatitis}

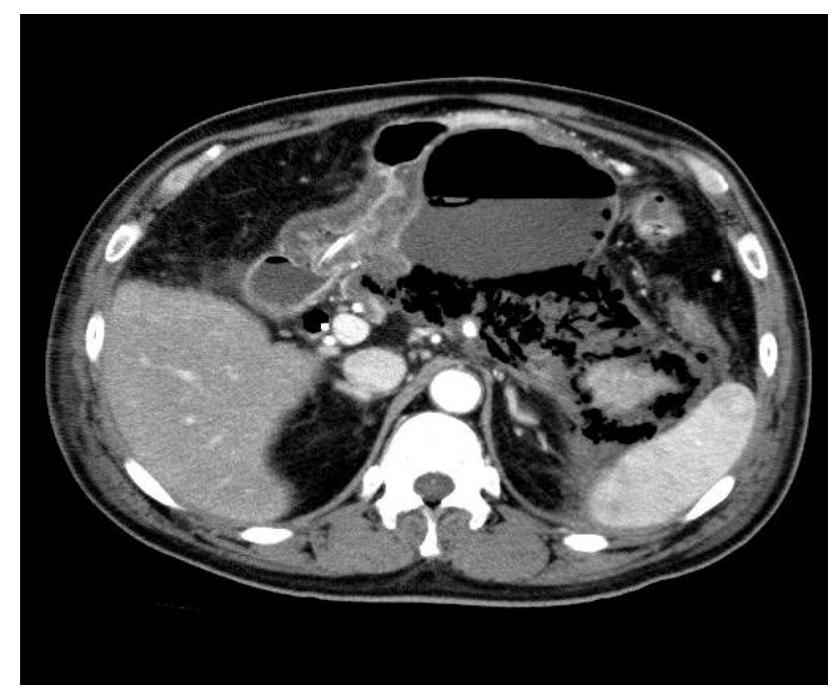

Received: 19 November 2015

Accepted: 6 December 2015

Published online: 22 December 2015

(C) Springer-Verlag Berlin Heidelberg and ESICM 2015

S. Hamada $\cdot$ N. Shime $(\varpi)$

National Hospital Organization Kyoto Medical Center, Kyoto,

Japan

e-mail: shime@koto.kpu-m.ac.jp

N. Shime

Hiroshima University, Hiroshima, Japan

A 63-year-old man with a 5-year history of diabetes was admitted to an intensive care unit (ICU) with epigastric pain, vomiting, and an increased serum amylase level of 5039 U/L. Computed tomography (CT) revealed acute gallstone pancreatitis, and the patient urgently underwent endoscopic retrograde biliary and pancreatic drainage. Contrast-enhanced CT on day 3 showed necrotic pancreatitis without gas. Progressive abdominal pain and fullness, however, subsequently developed. CT on day 12 depicted an exacerbation to emphysematous necrosis in the pancreas and a large amount of acute necrotic collection with air in the retroperitoneal space (Fig. 1), leading to a diagnosis of emphysematous pancreatitis. Endoscopic ultrasound-guided aspiration using two tubes for lavage and drainage through the stomach was performed. Gram-staining of the aspirates revealed gram-negative bacilli and gram-positive cocci/rods, while only Enterococcus faecalis was isolated by aerobic/anaerobic culture. His clinical course improved

Fig. $1 \mathrm{CT}$ showing an accumulation of air in the pancreas and retroperitoneal space with a necrotic collection

after administration of ampicillin and he was discharged from the ICU on day 17.

The two main origins of pancreatic gas are polymicrobial infections due to gas-forming organisms, such as Enterobacteriaceae or anaerobes, and fistulas between the pancreas and digestive tract [1]. In this case, however, neither the gas-forming organisms nor signs of fistula formation in CT and esophagogastroduodenoscopy were detected.

\title{
Reference
}

1. Kvinlaug K, Kriegler S, Moser M (2009)

Emphysematous pancreatitis: a less aggressive form of infected pancreatic necrosis? Pancreas 38:667-671. doi: 10.1097/MPA.0b013e3181a9f12a 